\title{
Nonlinear Heat Equation for Nonhomogeneous Anisotropic Materials: A Dual-Reciprocity Boundary Element Solution
}

\author{
Whye-Teong Ang, ${ }^{1}$ David L. Clements ${ }^{2}$ \\ ${ }^{1}$ Division of Engineering Mechanics, School of Mechanical and Aerospace \\ Engineering, Nanyang Technological University, Singapore 639798 \\ ${ }^{2}$ School of Mathematical Sciences, Faculty of Engineering, Computer and \\ Mathematical Sciences, The University of Adelaide, SA 5005, Australia
}

Received 31 October 2008; accepted 13 January 2009

Published online in Wiley InterScience (www.interscience.wiley.com).

DOI 10.1002/num.20452

\begin{abstract}
A dual-reciprocity boundary element method is presented for the numerical solution of initial-boundary value problems governed by a nonlinear partial differential equation for heat conduction in nonhomogeneous anisotropic materials. To assess the validity and accuracy of the method, some specific problems are solved. (c) 2009 Wiley Periodicals, Inc. Numer Methods Partial Differential Eq 000: 000-000, 2009
\end{abstract}

Keywords: boundary element method; heat conduction; nonhomogeneous materials; anisotropic materials; nonlinear partial heat equation

\section{INTRODUCTION}

In recent years, many nonlinear problems in physical and engineering science have been solved numerically using various boundary element approaches (see, e.g., Katsikadelis and Nerantzaki [1], Ang [2], Selvadurai [3], Chen, Hsiao, Chiu et al. [4], and Dehghan and Mirzaei [5]). One such problem which is of considerable interest to many researchers deals with heat conduction in solids with temperature dependent thermal conductivity. In earlier works on boundary element techniques for solving the nonlinear heat conduction problem, such as Kikuta et al. [6] and Goto and Suzuki [7], the solids are assumed to be thermally isotropic with material properties (density, specific heat capacity and thermal conductivity) which are functions of temperature alone. Extensions to thermally anisotropic solids with material properties that vary with temperature and spatial coordinates are given by Clements and Budhi [8] and Azis and Clements [9].

In the present article, we present a dual-reciprocity boundary element method for the problem of calculating the temperature in a nonhomogeneous anisotropic solid with density, specific heat capacity, and thermal conductivity which are functions of temperature and spatial coordinates. Specifically, the thermal conductivity coefficients are taken to be of the form considered in Azis 
and Clements [9] (i.e., as given in Eq. (2) in Section II later), but here (unlike in [9]) we do not require the function describing the spatial variation of the thermal conductivity [i.e., $g\left(x_{1}, x_{2}\right)$ ] to satisfy a particular partial differential equation. In [9], the boundary element approach in Goto and Suzuki [7], which approximates the domain integral arising in the formulation by discretizing the solution domain into many small cells, is exended to solve the governing nonlinear heat equation.

The boundary element approach presented here follows closely the works in Ang et al. [10] and Ang [11]. Firstly, guided by the analyses in Azis and Clements [9] and Ang et al. [10], we convert the governing nonlinear heat equation into a suitable integro-differential equation. The integro-differential equation involves an unknown function $\psi\left(x_{1}, x_{2}, t\right)$ and its first order partial derivative with respect to time $t$. It does not contain any partial derivative of $\psi$ with respect to the spatial coordinate $x_{i}$ and can be accurately solved for numerical values of $\psi$ by modifying the time-stepping dual-reciprocity boundary element procedure in Ang [11]. As in [11], $\partial \psi / \partial t$ is approximated in terms of a central difference formula, the integral over the boundary of the solution domain is discretized using discontinuous linear elements and the domain integral is approximated using the dual-reciprocity method. No meshing of the solution domain is required. The nonlinear term in the integrand of the integro-differential equation (i.e., the term containing $\partial \psi / \partial t$ multiplied to a given function of $x_{1}, x_{2}$, and $\left.\psi\right)$ is treated using a predictor-corrector (iterative) approach similar to that described in Ang and Ang [12].

The task of finding numerical values of $\psi$ at chosen points at consecutive time levels is eventually reduced to solving systems of linear algebraic equations. Once $\psi$ is determined, the temperature can be recovered by solving an algebraic equation which expresses $\psi$ as a function of the temperature and the spatial coordinates $x_{1}$ and $x_{2}$. To check its validity and to assess its accuracy, the numerical procedure presented here is applied to solve some specific problems with known solutions.

\section{THE PROBLEM}

With reference to a Cartesian coordinate system $O x_{1} x_{2} x_{3}$, consider a thermally nonhomogeneous anisotropic solid whose geometry does not vary along the $x_{3}$ axis. On the $O x_{1} x_{2}$ plane, the body occupies the region $R$ bounded by a simple closed curve $C$.

The temperature in the body is assumed to be independent of $x_{3}$. According to the classical theory of heat conduction, if there is no internal heat generation, the thermal behavior of the body is governed by the partial differential equation

$$
\frac{\partial}{\partial x_{i}}\left(k_{i j} \frac{\partial T}{\partial x_{j}}\right)=\rho c \frac{\partial T}{\partial t}
$$

where $k_{i j}$ are the thermal conductivity coefficients satisfying the symmetry relation $k_{i j}=k_{j i}$ and the strict inequality $k_{12}^{2}-k_{11} k_{22}<0, T\left(x_{1}, x_{2}, t\right)$ is the temperature at the point $\left(x_{1}, x_{2}\right)$ at time $t \geq 0$ and $\rho$ and $c$ are, respectively, the density and the specific heat capacity of the body. The usual Einstenian convention of summing over a repeated index is assumed here for Latin subscripts running from 1 to 2 .

The thermal conductivity coefficients $k_{i j}$ for the thermally nonhomogeneous anisotropic solid are taken to be of the form

$$
k_{i j}=\lambda_{i j} g\left(x_{1}, x_{2}\right) h(T),
$$

where $\lambda_{i j}$ are given positive constants such that $\lambda_{i j}=\lambda_{j i}$ and $\lambda_{12}^{2}-\lambda_{11} \lambda_{22}<0, g\left(x_{1}, x_{2}\right)$ is a given function such that $g\left(x_{1}, x_{2}\right)$ is positive and is at least twice partially differentiable with respect 
to $x_{1}$ and/or $x_{2}$ in $R \cup C$ and $h(T)$ is a given function which is integrable with respect to $T$. In general, the density $\rho$ and the specific heat capacity $c$ are suitably given functions which depend on $x_{1}, x_{2}$, and $T$.

The problem of interest here is to find the temperature $T\left(x_{1}, x_{2}, t\right)$ by solving (1) together with (2) in $R$ subject to to the initial-boundary conditions

$$
\begin{aligned}
T\left(x_{1}, x_{2}, 0\right) & =p\left(x_{1}, x_{2}\right) \text { for }\left(x_{1}, x_{2}\right) \in R, \\
T\left(x_{1}, x_{2}, t\right) & =u\left(x_{1}, x_{2}, t\right) \text { for }\left(x_{1}, x_{2}\right) \in C_{1} \text { and } t>0, \\
k_{i j} n_{i} \frac{\partial T}{\partial x_{j}} & =v\left(x_{1}, x_{2}, t\right) \text { for }\left(x_{1}, x_{2}\right) \in C_{2} \text { and } t>0,
\end{aligned}
$$

where $p, u$, and $v$ are suitably prescribed functions, $C_{1}$ and $C_{2}$ are nonintersecting curves such that $C_{1} \cup C_{2}=C$ and $\left[n_{1}\left(x_{1}, x_{2}\right), n_{2}\left(x_{1}, x_{2}\right)\right]$ is the unit normal outward vector to $R$ at the point $\left(x_{1}, x_{2}\right)$ on $C$.

\section{TRANSFORMED EQUATIONS}

If we apply the Kirchhoff's transformation

$$
\Theta\left(x_{1}, x_{2}, t\right)=\int h(T) d T \equiv K\left(T\left(x_{1}, x_{2}, t\right)\right)
$$

and assume that it can be inverted to give the temperature as $T=M(\Theta)$, then Eq. (1) together with (2) can be rewritten as

$$
\lambda_{i j} \frac{\partial}{\partial x_{i}}\left(g\left(x_{1}, x_{2}\right) \frac{\partial \Theta}{\partial x_{j}}\right)=S\left(x_{1}, x_{2}, \Theta\right) \frac{\partial \Theta}{\partial t},
$$

where

$$
S\left(x_{1}, x_{2}, \Theta\right)=\frac{\rho\left(x_{1}, x_{2}, M(\Theta)\right) c\left(x_{1}, x_{2}, M(\Theta)\right)}{h(M(\Theta))} .
$$

Furthermore, if we let

$$
\Theta\left(x_{1}, x_{2}, t\right)=\frac{1}{\sqrt{g\left(x_{1}, x_{2}\right)}} \psi\left(x_{1}, x_{2}, t\right),
$$

we find that (5) can be rewritten as

$$
\lambda_{i j} \frac{\partial^{2} \psi}{\partial x_{i} \partial x_{j}}=B\left(x_{1}, x_{2}\right) \psi+D\left(x_{1}, x_{2}, \psi\right) \frac{\partial \psi}{\partial t},
$$

where

$$
\begin{aligned}
B\left(x_{1}, x_{2}\right) & =\frac{1}{\sqrt{g\left(x_{1}, x_{2}\right)}} \lambda_{i j} \frac{\partial^{2}}{\partial x_{i} \partial x_{j}}\left[\sqrt{g\left(x_{1}, x_{2}\right)}\right] \\
D\left(x_{1}, x_{2}, \psi\right) & =\frac{1}{g\left(x_{1}, x_{2}\right)} S\left(x_{1}, x_{2}, \frac{1}{\sqrt{g\left(x_{1}, x_{2}\right)}} \psi\right) .
\end{aligned}
$$


The initial-boundary conditions in (3) can be rewritten as

$$
\begin{aligned}
\psi\left(x_{1}, x_{2}, 0\right)= & \sqrt{g\left(x_{1}, x_{2}\right)} K\left(p\left(x_{1}, x_{2}\right)\right) \text { for }\left(x_{1}, x_{2}\right) \in R, \\
\psi\left(x_{1}, x_{2}, t\right)= & \sqrt{g\left(x_{1}, x_{2}\right)} K\left(u\left(x_{1}, x_{2}, t\right)\right) \\
& \text { for }\left(x_{1}, x_{2}\right) \in C_{1} \text { and } t>0, \\
q\left(x_{1}, x_{2}, t\right)= & f\left(x_{1}, x_{2}, t\right) \psi\left(x_{1}, x_{2}, t\right)+\frac{1}{\sqrt{g\left(x_{1}, x_{2}\right)}} v\left(x_{1}, x_{2}, t\right) \\
& \text { for }\left(x_{1}, x_{2}\right) \in C_{2} \text { and } t>0,
\end{aligned}
$$

where

$$
\begin{aligned}
f\left(x_{1}, x_{2}\right) & =\frac{1}{2} \frac{1}{g\left(x_{1}, x_{2}\right)} n_{i} \lambda_{i j} \frac{\partial g}{\partial x_{j}} \\
q\left(x_{1}, x_{2}, t\right) & =n_{i} \lambda_{i j} \frac{\partial \psi}{\partial x_{j}} .
\end{aligned}
$$

Following closely the approach in Ang et al. [10] and Ang [11], we recast (8) into an integro-differential equation given by

$$
\begin{aligned}
& \gamma\left(\xi_{1}, \xi_{2}\right) \psi\left(\xi_{1}, \xi_{2}, t\right) \\
& =\iint_{R} \Phi\left(x_{1}, x_{2}, \xi_{1}, \xi_{2}\right)\left[B\left(x_{1}, x_{2}\right) \psi\left(x_{1}, x_{2}, t\right)\right. \\
& \left.\quad+D\left(x_{1}, x_{2}, \psi\left(x_{1}, x_{2}, t\right)\right) \frac{\partial}{\partial t}\left\{\psi\left(x_{1}, x_{2}, t\right)\right\}\right] d x_{1} d x_{2} \\
& \quad+\oint_{C}\left[\Gamma\left(x_{1}, x_{2}, \xi_{1}, \xi_{2}\right) \psi\left(x_{1}, x_{2}, t\right)-\Phi\left(x_{1}, x_{2}, \xi_{1}, \xi_{2}\right) q\left(x_{1}, x_{2}, t\right)\right] d s\left(x_{1}, x_{2}\right),
\end{aligned}
$$

where $\gamma\left(\xi_{1}, \xi_{2}\right)=0$ if $\left(\xi_{1}, \xi_{2}\right) \notin R \cup C, \gamma\left(\xi_{1}, \xi_{2}\right)=1$ if $\left(\xi_{1}, \xi_{2}\right) \in R, 0<\gamma\left(\xi_{1}, \xi_{2}\right)<1$ if $\left(\xi_{1}, \xi_{2}\right) \in C\left[\gamma\left(\xi_{1}, \xi_{2}\right)=1 / 2\right.$ if $\left(\xi_{1}, \xi_{2}\right)$ lies on a smooth part of $C$ and

$$
\begin{aligned}
\Phi\left(x_{1}, x_{2}, \xi_{1}, \xi_{2}\right) & =\frac{1}{2 \pi \sqrt{\lambda_{11} \lambda_{22}-\lambda_{12}^{2}}} \operatorname{Re}\left\{\ln \left(x_{1}-\xi_{1}+\tau\left[x_{2}-\xi_{2}\right]\right)\right\}, \\
\Gamma\left(x_{1}, x_{2}, \xi_{1}, \xi_{2}\right) & =\frac{1}{2 \pi \sqrt{\lambda_{11} \lambda_{22}-\lambda_{12}^{2}}} \operatorname{Re}\left\{\frac{L\left(x_{1}, x_{2}\right)}{\left(x_{1}-\xi_{1}+\tau\left[x_{2}-\xi_{2}\right]\right)}\right\}, \\
L\left(x_{1}, x_{2}\right) & =\left(\lambda_{11}+\tau \lambda_{12}\right) n_{1}\left(x_{1}, x_{2}\right)+\left(\lambda_{21}+\tau \lambda_{22}\right) n_{2}\left(x_{1}, x_{2}\right), \\
\tau & =\frac{-\lambda_{12}+i \sqrt{\lambda_{11} \lambda_{22}-\lambda_{12}^{2}}}{\lambda_{22}} \quad(i=\sqrt{-1}) .
\end{aligned}
$$

Note that Re denotes the real part of a complex number.

Section IV describes a dual-reciprocity boundary element approach for finding $\psi\left(x_{1}, x_{2}, t\right)$ numerically from (12) together with (10). Once $\psi\left(x_{1}, x_{2}, t\right)$ is determined, the temperature $T\left(x_{1}, x_{2}, t\right)$ may be obtained by inverting the Kirchhoff's transformation in (4). 


\section{DUAL-RECIPROCITY BOUNDARY ELEMENT METHOD}

For the dual-reciprocity boundary element method, we discretize the curve $C_{1}$ into $N_{1}$ straight line elements denoted by $C^{(1)}, C^{(2)}, \ldots, C^{\left(N_{1}-1\right)}$ and $C^{\left(N_{1}\right)}$ and $C_{2}$ into $C^{\left(N_{1}+1\right)}, C^{\left(N_{1}+2\right)}, \ldots, C^{\left(N_{1}+N_{2}-1\right)}$, and $C^{\left(N_{1}+N_{2}\right)}$. Note that $C_{1}$ and $C_{2}$ are parts of the boundary $C$ [refer to the boundary conditions in (3) or (10)]. Thus, the total number of boundary elements is given by $N=N_{1}+N_{2}$. The element $C^{(m)}(m=1,2, \ldots, N)$ has length $\ell^{(m)}$ and its starting and ending points are given by $\left(a_{1}^{(m)}, a_{2}^{(m)}\right)$ and $\left(b_{1}^{(m)}, b_{2}^{(m)}\right)$ respectively.

For an accurate approximation, $\psi$ and $q$ in (12) on the boundary are approximated using discontinuous linear boundary elements as outlined in París and Cañas [13]. For the discontinuous linear boundary elements, two points $\left(\eta_{1}^{(m)}, \eta_{2}^{(m)}\right)$ and $\left(\eta_{1}^{(N+m)}, \eta_{2}^{(N+m)}\right)$ on $C^{(m)}$ are chosen as given by

$$
\left.\begin{array}{c}
\eta_{i}^{(m)}=a_{i}^{(m)}+r\left(b_{i}^{(m)}-a_{i}^{(m)}\right) \\
\eta_{i}^{(N+m)}=b_{i}^{(m)}-r\left(b_{i}^{(m)}-a_{i}^{(m)}\right)
\end{array}\right\} \text { for a given } r \in\left(0, \frac{1}{2}\right) .
$$

Note that $r$ is a selected constant between 0 and $1 / 2$. It may typically be chosen to be $1 / 4$.

If $\psi$ is given by $\psi^{(m)}(t)$ and $\psi^{(N+m)}(t)$ at $\left(\eta_{1}^{(m)}, \eta_{2}^{(m)}\right)$, and $\left(\eta_{1}^{(N+m)}, \eta_{2}^{(N+m)}\right)$ respectively, then we make the approximation:

$$
\psi\left(x_{1}, x_{2}, t\right) \simeq\left[1-d^{(m)}\left(x_{1}, x_{2}\right)\right] \psi^{(m)}(t)+d^{(m)}\left(x_{1}, x_{2}\right) \psi^{(N+m)}(t) \text { for }\left(x_{1}, x_{2}\right) \in C^{(m)},
$$

where

$$
d^{(m)}\left(x_{1}, x_{2}\right)=\frac{\sqrt{\left(x_{1}-a_{1}^{(m)}\right)^{2}+\left(x_{2}-a_{2}^{(m)}\right)^{2}}-r \ell^{(m)}}{(1-2 r) \ell^{(m)}} .
$$

Similarly, for $q$, if it is given by $q^{(m)}(t)$ and $q^{(N+m)}(t)$ at $\left(\eta_{1}^{(m)}, \eta_{2}^{(m)}\right)$ and $\left(\eta_{1}^{(N+m)}, \eta_{2}^{(N+m)}\right)$, respectively, then

$$
q\left(x_{1}, x_{2}, t\right) \simeq\left[1-d^{(m)}\left(x_{1}, x_{2}\right)\right] q^{(m)}(t)+d^{(m)}\left(x_{1}, x_{2}\right) q^{(N+m)}(t) \text { for }\left(x_{1}, x_{2}\right) \in C^{(m)} .
$$

With (15) and (17), the integro-differential equation in (12) can be approximately written as

$$
\begin{aligned}
\gamma\left(\xi_{1}, \xi_{2}\right) \psi\left(\xi_{1}, \xi_{2}, t\right)= & \iint_{R} \Phi\left(x_{1}, x_{2}, \xi_{1}, \xi_{2}\right)\left[B\left(x_{1}, x_{2}\right) \psi\left(x_{1}, x_{2}, t\right)\right. \\
& \left.+D\left(x_{1}, x_{2}, \psi\left(x_{1}, x_{2}, t\right)\right) \frac{\partial}{\partial t}\left\{\psi\left(x_{1}, x_{2}, t\right)\right\}\right] d x_{1} d x_{2} \\
& +\sum_{m=1}^{N}\left\{\psi^{(m)}(t) \Omega_{1}^{(m)}\left(\xi_{1}, \xi_{2}\right)+\psi^{(N+m)}(t) \Omega_{2}^{(m)}\left(\xi_{1}, \xi_{2}\right)\right\} \\
& -\sum_{m=1}^{N}\left\{q^{(m)}(t) \Omega_{3}^{(m)}\left(\xi_{1}, \xi_{2}\right)+q^{(N+m)}(t) \Omega_{4}^{(m)}\left(\xi_{1}, \xi_{2}\right)\right\}
\end{aligned}
$$


where

$$
\begin{aligned}
& \Omega_{1}^{(m)}\left(\xi_{1}, \xi_{2}\right)=\int_{C^{(m)}}\left[1-d^{(m)}\left(x_{1}, x_{2}\right)\right] \Gamma\left(x_{1}, x_{2}, \xi_{1}, \xi_{2}\right) d s\left(x_{1}, x_{2}\right), \\
& \Omega_{2}^{(m)}\left(\xi_{1}, \xi_{2}\right)=\int_{C^{(m)}} d^{(m)}\left(x_{1}, x_{2}\right) \Gamma\left(x_{1}, x_{2}, \xi_{1}, \xi_{2}\right) d s\left(x_{1}, x_{2}\right), \\
& \Omega_{3}^{(m)}\left(\xi_{1}, \xi_{2}\right)=\int_{C^{(m)}}\left[1-d^{(m)}\left(x_{1}, x_{2}\right)\right] \Phi\left(x_{1}, x_{2}, \xi_{1}, \xi_{2}\right) d s\left(x_{1}, x_{2}\right), \\
& \Omega_{4}^{(m)}\left(\xi_{1}, \xi_{2}\right)=\int_{C^{(m)}} d^{(m)}\left(x_{1}, x_{2}\right) \Phi\left(x_{1}, x_{2}, \xi_{1}, \xi_{2}\right) d s\left(x_{1}, x_{2}\right) .
\end{aligned}
$$

Exact formulae for calculating the line integrals over $C^{(m)}$ in (19) are given in Ang [11].

To treat the domain integral in (18) using the dual-reciprocity method, we select $P$ well spaced out points in the interior of the domain $R$. These interior points are denoted by $\left(\eta_{1}^{(2 N+1)}, \eta_{2}^{(2 N+1)}\right)$, $\left(\eta_{1}^{(2 N+2)}, \eta_{2}^{(2 N+2)}\right), \ldots,\left(\eta_{1}^{(2 N+P-1)}, \eta_{2}^{(2 N+P-1)}\right)$, and $\left(\eta_{1}^{(2 N+P)}, \eta_{2}^{(2 N+P)}\right)$. We define $\psi^{(2 N+j)}(t)=$ $\psi\left(\eta_{1}^{(2 N+1)}, \eta_{2}^{(2 N+1)}, t\right)$ for $j=1,2, \ldots, P$. Following the procedure detailed in Ang et al. [10] and Ang [11], we then approximate the domain integral using

$$
\begin{aligned}
& \iint_{R} \Phi\left(x_{1}, x_{2}, \xi_{1}, \xi_{2}\right)\left[B\left(x_{1}, x_{2}\right) \psi\left(x_{1}, x_{2}, t\right)+D\left(x_{1}, x_{2}, \psi\left(x_{1}, x_{2}, t\right)\right) \frac{\partial}{\partial t}\left\{\psi\left(x_{1}, x_{2}, t\right)\right\}\right] d x_{1} d x_{2} \\
& \simeq \sum_{k=1}^{2 N+P}\left[B\left(\eta_{1}^{(k)}, \eta_{2}^{(k)}\right) \psi^{(k)}(t)+D\left(\eta_{1}^{(k)}, \eta_{2}^{(k)}, \psi^{(k)}(t)\right) \frac{d}{d t}\left\{\psi^{(k)}(t)\right\}\right] \sum_{j=1}^{2 N+P} \chi^{(k j)} \Psi^{(j)}\left(\xi_{1}, \xi_{2}\right),
\end{aligned}
$$

where

$$
\begin{aligned}
& \sum_{k=1}^{2 N+P} \sigma^{(j)}\left(\eta_{1}^{(k)}, \eta_{2}^{(k)}\right) \chi^{(k m)}= \begin{cases}1 & \text { if } j=m \\
0 & \text { if } j \neq m\end{cases} \\
& \text { for } j, m=1,2, \ldots, 2 N+P \text {, } \\
& \sigma^{(j)}\left(x_{1}, x_{2}\right)=1+\left(\left[x_{1}-\eta_{1}^{(j)}+\operatorname{Re}\{\tau\}\left\{x_{2}-\eta_{2}^{(j)}\right\}\right]^{2}+\left[\operatorname{Im}\{\tau\}\left\{x_{2}-\eta_{2}^{(j)}\right\}\right]^{2}\right) \\
& +\left(\left[x_{1}-\eta_{1}^{(j)}+\operatorname{Re}\{\tau\}\left\{x_{2}-\eta_{2}^{(j)}\right\}\right]^{2}+\left[\operatorname{Im}\{\tau\}\left\{x_{2}-\eta_{2}^{(j)}\right\}\right]^{2}\right)^{3 / 2} \\
& \text { for } j=1,2, \ldots, 2 N+P \text {, } \\
& \Psi^{(j)}\left(\xi_{1}, \xi_{2}\right)=\gamma\left(\xi_{1}, \xi_{2}\right) \theta^{(j)}\left(\xi_{1}, \xi_{2}\right)-\int_{C} \Phi\left(x_{1}, x_{2}, \xi_{1}, \xi_{2}\right) \beta^{(j)}\left(x_{1}, x_{2}\right) d s\left(x_{1}, x_{2}\right)
\end{aligned}
$$




$$
\begin{aligned}
& -\int_{C} \theta^{(j)}\left(x_{1}, x_{2}\right) \Gamma\left(x_{1}, x_{2}, \xi_{1}, \xi_{2}\right) d s\left(x_{1}, x_{2}\right) \\
& \text { for } j=1,2, \ldots, 2 N+P \text {, } \\
& \left(\frac{\lambda_{11} \lambda_{22}-\lambda_{12}^{2}}{\lambda_{22}}\right) \theta^{(j)}\left(x_{1}, x_{2}\right)=\frac{1}{4}\left(\left[x_{1}-\eta_{1}^{(j)}+\operatorname{Re}\{\tau\}\left\{x_{2}-\eta_{2}^{(j)}\right\}\right]^{2}+\left[\operatorname{Im}\{\tau\}\left\{x_{2}-\eta_{2}^{(j)}\right\}\right]^{2}\right) \\
& +\frac{1}{16}\left(\left[x_{1}-\eta_{1}^{(j)}+\operatorname{Re}\{\tau\}\left\{x_{2}-\eta_{2}^{(j)}\right\}\right]^{2}+\left[\operatorname{Im}\{\tau\}\left\{x_{2}-\eta_{2}^{(j)}\right\}\right]^{2}\right)^{2} \\
& +\frac{1}{25}\left(\left[x_{1}-\eta_{1}^{(j)}+\operatorname{Re}\{\tau\}\left\{x_{2}-\eta_{2}^{(j)}\right\}\right]^{2}+\left[\operatorname{Im}\{\tau\}\left\{x_{2}-\eta_{2}^{(j)}\right\}\right]^{2}\right)^{5 / 2}, \\
& \beta^{(j)}\left(x_{1}, x_{2}\right)=-\sum_{i=1}^{2} \sum_{k=1}^{2} \lambda_{i k} n_{i}\left(x_{1}, x_{2}\right) \frac{\partial \theta^{(j)}}{\partial x_{k}} .
\end{aligned}
$$

Note that $\sigma^{(j)}\left(x_{1}, x_{2}\right)$ are the radial basis functions used in Ang et al. [10] for anisotropic media. For $\lambda_{i j}=\delta_{i j}$ (Kronecker-delta), we find that $\tau=i$ and $\sigma^{(j)}\left(x_{1}, x_{2}\right)$ given above reduces to give the local interpolating functions suggested by Zhang and Zhu [14].

The functions $\Psi^{(j)}\left(\xi_{1}, \xi_{2}\right)$ in (21) are expressed in terms of line integrals over the boundary $C$ and can easily be computed approximately using

$$
\begin{aligned}
\Psi^{(j)}\left(\xi_{1}, \xi_{2}\right) \simeq & \gamma\left(\xi_{1}, \xi_{2}\right) \theta^{(j)}\left(\xi_{1}, \xi_{2}\right) \\
& -\sum_{m=1}^{N}\left\{\beta^{(j)}\left(\eta_{1}^{(m)}, \eta_{2}^{(m)}\right) \Omega_{3}^{(m)}\left(\xi_{1}, \xi_{2}\right)\right. \\
& -\beta^{(j)}\left(\eta_{1}^{(N+m)}, \eta_{2}^{(N+m)}\right) \Omega_{4}^{(m)}\left(\xi_{1}, \xi_{2}\right) \\
& -\theta^{(j)}\left(\eta_{1}^{(m)}, \eta_{2}^{(m)}\right) \Omega_{1}^{(m)}\left(\xi_{1}, \xi_{2}\right) \\
& \left.-\theta^{(j)}\left(\eta_{1}^{(N+m)}, \eta_{2}^{(N+m)}\right) \Omega_{2}^{(m)}\left(\xi_{1}, \xi_{2}\right)\right\} .
\end{aligned}
$$

No discretization of the solution domain into elements is therefore needed in computing the domain integral in (18).

If we make the approximation

$$
\begin{aligned}
\psi^{(k)}(t) & \simeq \frac{1}{2}\left[\psi^{(k)}\left(t+\frac{1}{2} \Delta t\right)+\psi^{(k)}\left(t-\frac{1}{2} \Delta t\right)\right], \\
\frac{d}{d t}\left[\psi^{(k)}(t)\right] & \simeq \frac{\psi^{(k)}\left(t+\frac{1}{2} \Delta t\right)-\psi^{(k)}\left(t-\frac{1}{2} \Delta t\right)}{\Delta t},
\end{aligned}
$$


let $\left(\xi_{1}, \xi_{2}\right)$ in (18) be given in turn by $\left(\eta_{1}^{(p)}, \eta_{2}^{(p)}\right)$ for $p=1,2, \ldots, 2 N+P$, and use the boundary conditions in (10), we obtain

$$
\begin{aligned}
& \left(\frac{1}{2} \alpha^{(p)}\left[\psi^{(p)}\left(t+\frac{1}{2} \Delta t\right)+\psi^{(p)}\left(t-\frac{1}{2} \Delta t\right)\right]+\left(1-\alpha^{(p)}\right) R\left(\eta_{1}^{(p)}, \eta_{2}^{(p)}, t\right)\right) \gamma\left(\eta_{1}^{(p)}, \eta_{2}^{(p)}\right) \\
& =\sum_{k=1}^{2 N+P}\left[\frac{1}{2} B\left(\eta_{1}^{(k)}, \eta_{2}^{(k)}\right)\left\{\psi^{(k)}\left(t+\frac{1}{2} \Delta t\right)+\psi^{(k)}\left(t-\frac{1}{2} \Delta t\right)\right\}\right. \\
& \left.+\frac{1}{\Delta t} E^{(k)}(t)\left\{\psi^{(k)}\left(t+\frac{1}{2} \Delta t\right)-\psi^{(k)}\left(t-\frac{1}{2} \Delta t\right)\right\}\right] \alpha^{(k)} \mu^{(k p)} \\
& +\sum_{k=1}^{2 N}\left(1-\alpha^{(k)}\right) \mu^{(k p)}\left(B\left(\eta_{1}^{(k)}, \eta_{2}^{(k)}\right) R\left(\eta_{1}^{(k)}, \eta_{2}^{(k)}, t\right)+E^{(k)}(t) \frac{d}{d t}\left\{R\left(\eta_{1}^{(k)}, \eta_{2}^{(k)}, t\right)\right\}\right) \\
& +\sum_{m=1}^{N_{1}}\left\{\Omega_{1}^{(m)}\left(\eta_{1}^{(p)}, \eta_{2}^{(p)}\right) R\left(\eta_{1}^{(m)}, \eta_{2}^{(m)}, t\right)+\Omega_{2}^{(m)}\left(\eta_{1}^{(p)}, \eta_{2}^{(p)}\right) R\left(\eta_{1}^{(N+m)}, \eta_{2}^{(N+m)}, t\right)\right\} \\
& +\sum_{m=N_{1}+1}^{N}\left\{\frac{1}{2}\left(\Omega_{1}^{(m)}\left(\eta_{1}^{(p)}, \eta_{2}^{(p)}\right)-\Omega_{3}^{(m)}\left(\eta_{1}^{(p)}, \eta_{2}^{(p)}\right) f\left(\eta_{1}^{(m)}, \eta_{2}^{(m)}\right)\right)\right. \\
& \times\left[\psi^{(m)}\left(t+\frac{1}{2} \Delta t\right)+\psi^{(m)}\left(t-\frac{1}{2} \Delta t\right)\right] \\
& +\frac{1}{2}\left(\Omega_{2}^{(m)}\left(\eta_{1}^{(p)}, \eta_{2}^{(p)}\right)-\Omega_{4}^{(m)}\left(\eta_{1}^{(p)}, \eta_{2}^{(p)}\right) f\left(\eta_{1}^{(N+m)}, \eta_{2}^{(N+m)}\right)\right) \\
& \left.\times\left[\psi^{(N+m)}\left(t+\frac{1}{2} \Delta t\right)+\psi^{(N+m)}\left(t-\frac{1}{2} \Delta t\right)\right]\right\} \\
& -\sum_{m=1}^{N_{1}}\left\{q^{(m)}(t) \Omega_{3}^{(m)}\left(\eta_{1}^{(p)}, \eta_{2}^{(p)}\right)+q^{(N+m)}(t) \Omega_{4}^{(m)}\left(\eta_{1}^{(p)}, \eta_{2}^{(p)}\right)\right\} \\
& -\sum_{m=N_{1}+1}^{N}\left\{\Omega_{3}^{(m)}\left(\eta_{1}^{(p)}, \eta_{2}^{(p)}\right) \frac{1}{\sqrt{g\left(\eta_{1}^{(m)}, \eta_{2}^{(m)}\right)}} v\left(\eta_{1}^{(m)}, \eta_{2}^{(m)}, t\right)\right] \\
& \left.+\Omega_{4}^{(m)}\left(\eta_{1}^{(p)}, \eta_{2}^{(p)}\right) \frac{1}{\sqrt{g\left(\eta_{1}^{(N+m)}, \eta_{2}^{(N+m)}\right)}} v\left(\eta_{1}^{(N+m)}, \eta_{2}^{(N+m)}, t\right)\right\} \\
& \text { for } p=1,2, \ldots, 2 N+P \text {, }
\end{aligned}
$$

where

$$
\begin{aligned}
E^{(k)}(t) & =D\left(\eta_{1}^{(k)}, \eta_{2}^{(k)}, \psi^{(k)}(t)\right), \\
\mu^{(k p)} & =\sum_{j=1}^{2 N+P} \chi^{(k j)} \Psi^{(j)}\left(\eta_{1}^{(p)}, \eta_{2}^{(p)}\right),
\end{aligned}
$$




$$
\begin{aligned}
\alpha^{(k)} & = \begin{cases}0 & \text { for } 1 \leq k \leq N_{1} \text { or } N+1 \leq k \leq N+N_{1}, \\
1 & \text { otherwise, }\end{cases} \\
R\left(\eta_{1}, \eta_{2}, t\right) & =\sqrt{g\left(\eta_{1}, \eta_{2}\right)} K\left(u\left(\eta_{1}, \eta_{2}, t\right)\right) .
\end{aligned}
$$

If $E^{(n)}(t)$ and $\psi^{(n)}\left(t-\frac{1}{2} \Delta t\right)$ are assumed known for $n=1,2, \ldots, 2 N+P$ then (24) constitutes a system of $2 N+P$ linear algebraic equations containing $2 N+P$ unknowns given by $q^{(m)}(t)$ and $q^{(N+m)}(t)$ for $m=1,2, \ldots, N_{1}, \psi^{(m)}\left(t+\frac{1}{2} \Delta t\right)$ and $\psi^{(N+m)}\left(t+\frac{1}{2} \Delta t\right)$ for $m=N_{1}+1$, $N_{1}+2, \ldots, N_{1}+N_{2}$, and $\psi^{(p)}\left(t+\frac{1}{2} \Delta t\right)$ for $p=2 N+1,2 N+2, \ldots, 2 N+P$. (Note that $N_{1}+N_{2}=N$.) The unknowns can be determined numerically by repeating the steps below until the numerical values of $\psi$ at the selected points are obtained at the desired time level.

1. From the initial condition given in (10), compute the values of $\psi^{(n)}(0)$ for $n=1,2, \ldots, 2 N+$ $P$. Choose a small positive time-step $\Delta t$. Set the integer $J=0$. Go to Step 2 .

2. Estimate the values of $E^{(n)}(J \Delta t)$ using the latest known values of $\psi^{(n)}(J \Delta t)$, that is, $E^{(n)}(J \Delta t) \simeq D\left(\eta_{1}^{(n)}, \eta_{2}^{(n)}, \psi^{(n)}(J \Delta t)\right)$. Go to Step 3 .

3. Using the latest known values of $E^{(n)}(J \Delta t)$ and $\psi^{(n)}(J \Delta t)$, let $t=\left(J+\frac{1}{2}\right) \Delta t$ in (24) to set up a system of linear algebraic equations and solve for the unknowns $q^{(m)}\left(\left(J+\frac{1}{2}\right) \Delta t\right)$ and $q^{(N+m)}\left(\left(J+\frac{1}{2}\right) \Delta t\right)$ for $m=1,2, \ldots, N_{1}, \psi^{(j)}((J+1) \Delta t)$ and $\psi^{(N+j)}((J+1) \Delta t)$ for $j=N_{1}+1, N_{1}+2, \ldots, N_{1}+N_{2}$, and $\psi^{(p)}((J+1) \Delta t)$ for $p=2 N+1,2 N+2, \ldots, 2 N+P$. Go to Step 4.

4. Use the latest known values of $\psi^{(n)}((J+1) \Delta t)$ obtained in Step 3 above to compute $\psi^{(n)}\left(\left(J+\frac{1}{2}\right) \Delta t\right)=\frac{1}{2}\left[\psi^{(n)}((J+1) \Delta t)+\psi^{(n)}(J \Delta t)\right]$ for $n=1,2, \ldots, 2 N+P$. Recalculate $E^{(n)}(J \Delta t)$ using $E^{(n)}(J \Delta t) \simeq D\left(\eta_{1}^{(n)}, \eta_{2}^{(n)}, \psi^{(n)}\left(\left(J+\frac{1}{2}\right) \Delta t\right)\right)$. Check whether the newly obtained values of $E^{(n)}(J \Delta t)$ agree with the previous values to within a specified number of significant figures. If the required convergence is not achieved, go to Step 3. Otherwise, increase the current value of $J$ by 1 and go to Step 2 .

\section{SPECIFIC NUMERICAL EXAMPLES}

To assess the validity and accuracy of the dual-reciprocity boundary element procedure outlined earlier, it is applied to solve some specific problems here.

Problem 1. The thermal conductivity, density and specific heat capacity of the material are taken to be given by $k_{i j}=\delta_{i j}(1+T), \rho=1$ and $c=1+\frac{1}{2} T$ respectively. Note that $\delta_{i j}$ is the Kronecker-delta. The solution domain $R$ is taken to be rectangular in shape, defined by $0<x_{1}<1,0<x_{2}<1 / 5$. Refer to Fig. 1 . The initial-boundary conditions are given by

$$
\begin{aligned}
& T\left(x_{1}, x_{2}, 0\right)=0 \text { for }\left(x_{1}, x_{2}\right) \in R, \\
& T\left(0, x_{2}, t\right)=1 \text { for } 0<x_{2}<\frac{1}{5} \text { and } t>0, \\
& T\left(1, x_{2}, t\right)=0 \text { for } 0<x_{2}<\frac{1}{5} \text { and } t>0,
\end{aligned}
$$




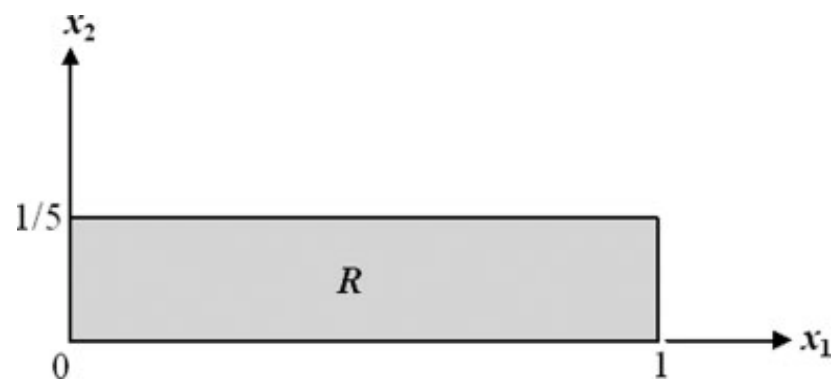

FIG. 1. A sketch of the solution domain for Problem 1.

$$
\begin{gathered}
\left.k_{i j} n_{i} \frac{\partial T}{\partial x_{j}}\right|_{x_{2}=0}=0 \text { for } 0<x_{1}<1 \text { and } t>0, \\
\left.k_{i j} n_{i} \frac{\partial T}{\partial x_{j}}\right|_{x_{2}=1 / 5}=0 \text { for } 0<x_{1}<1 \text { and } t>0 .
\end{gathered}
$$

For this specific problem, $\lambda_{i j}=\delta_{i j}, h(T)=1+T$ and $g\left(x_{1}, x_{2}\right)=1$. Hence, from (4) and (7), we may write $\psi / \sqrt{g}=T+\frac{1}{2} T^{2}$. Solving for $T$ and taking the positive square root of the determinant yields $T=-1+\sqrt{2 \psi / \sqrt{g}+1}$. It follows that the partial differential equation to solve is given by

$$
\frac{\partial^{2} \psi}{\partial x_{i} \partial x_{i}}=\frac{1+\sqrt{(2 \psi / \sqrt{g}+1)}}{2 \sqrt{(2 \psi / \sqrt{g}+1)}} \frac{\partial \psi}{\partial t},
$$

and the initial-boundary conditions are

$$
\begin{aligned}
\psi\left(x_{1}, x_{2}, 0\right) & =0 \text { for }\left(x_{1}, x_{2}\right) \in R \\
\psi\left(0, x_{2}, t\right) & =\frac{3}{2} \text { for } 0<x_{2}<\frac{1}{5} \text { and } t>0 \\
\psi\left(1, x_{2}, t\right) & =0 \text { for } 0<x_{2}<\frac{1}{5} \text { and } t>0 \\
q\left(x_{1}, 0, t\right) & =0 \text { for } 0<x_{1}<1 \text { and } t>0 \\
q\left(x_{1}, \frac{1}{5}, t\right) & =0 \text { for } 0<x_{1}<1 \text { and } t>0
\end{aligned}
$$

The sides of the rectangular domain are discretized into $N$ equal length boundary elements. The interior collocation points are given by $\left(m /\left(M_{1}+1\right), n /\left[5\left(M_{2}+1\right)\right]\right)$ for $m=1,2, \ldots, M_{1}$ and $n=1,2, \ldots, M_{2}$. The parameter $r$ in (14) is chosen to be $1 / 4$ and the time-step $\Delta t$ by $1 /\left(J_{0}+\frac{1}{2}\right)$, where $J_{0}$ is a selected positive integer. The time-stepping dual-reciprocity boundary element method (DRBEM) is applied to compute $\psi\left(x_{1}, x_{2}, t\right)$ numerically. Specifically, in following the steps outlined in Section IV, we take $J=0,1,2, \ldots, J_{0}$ (consecutively) to compute numerically the values of $\psi$ at the chosen collocation points at consecutive time levels until we obtain $\psi^{(n)}(1)=\frac{1}{2}\left[\psi^{(n)}\left(\left(J_{0}+1\right) /\left(J_{0}+\frac{1}{2}\right)\right)+\psi^{(n)}\left(J_{0} /\left(J_{0}+\frac{1}{2}\right)\right)\right]$ for $n=1,2, \ldots, 2 N+M_{1} M_{2}$. For each value of $J$, that is, at each time level, the final numerical values of $\psi^{(n)}\left(\left(J+\frac{1}{2}\right) /\left(J_{0}+\frac{1}{2}\right)\right)$ are obtained after iterating 3 times to and fro the last two steps in Section IV. The numerical values 
TABLE I. Numerical values of $T$ at time $t=1$ at selected interior points.

\begin{tabular}{cccc}
\hline$\left(x_{1}, x_{2}\right)$ & Azis and Clements & Goto and Suzuki & Present DRBEM \\
\hline$(0.20,0.10)$ & 0.8398 & 0.8439 & 0.8448 \\
$(0.40,0.10)$ & 0.6664 & 0.6733 & 0.6738 \\
$(0.60,0.10)$ & 0.4754 & 0.4832 & 0.4826 \\
$(0.80,0.10)$ & 0.2589 & 0.2649 & 0.2649 \\
\hline
\end{tabular}

of $\psi^{(n)}(1)$ obtained using $N=96$ (each element of length 0.125 units), $M_{1}=9, M_{2}=7$ (63 interior collocation points $)$ and $J_{0}=10(\Delta t=2 / 21 \simeq 0.0952)$ are used to compute the temperature at the collocation points $(0.20,0.10),(0.40,0.10),(0.60,0.10)$ and $(0.80,0.10)$ at time $t=1$. The numerical values of the temperature are compared with those given in Azis and Clements [9] and Goto and Suzuki [7] in Table I. (In Refs. [7] and [9], the temperature is calculated using a different boundary element approach.) The three sets of numerical values appear to be reasonably close to one another, but our results here seem to be closer to those given by Goto and Suzuki [7] than Azis and Clements [9].

Problem 2. In a particular problem considered in Azis and Clements [9], the thermal conductivity, density and specific heat capacity are taken to be such that

$$
\left[k_{i j}\right]=\left(1+\frac{1}{10} x_{1}\right)^{2}\left[\begin{array}{ll}
3 & 1 \\
1 & 4
\end{array}\right] \text { and } \rho c=\frac{9}{2 T}\left(1+\frac{1}{10} x_{1}\right) .
$$

Here $\lambda_{11}=3, \lambda_{12}=\lambda_{21}=1, \lambda_{22}=4, g\left(x_{1}, x_{2}\right)=\left(1+\frac{1}{10} x_{1}\right)^{2}$, and $h(T)=1$.

With $k_{i j}$ and $\rho c$ as given above, it may be verified that a particular solution of (1) is given by

$$
T\left(x_{1}, x_{2}, t\right)=\frac{1-\frac{1}{4}\left(x_{1}+x_{2}\right)^{2}}{(1+t)\left(1+\frac{1}{10} x_{1}\right)} .
$$

Proceeding as in Azis and Clements [9], we use the above particular solution to generate boundary data for $T$ and $k_{i j} n_{i} \partial T / \partial x_{j}$, respectively, on the horizontal and vertical sides of the square domain $0<x_{1}<1,0<x_{2}<1$.

For this particular problem, $\psi\left(x_{1}, x_{2}, t\right)=\left(1+\frac{1}{10} x_{1}\right) T\left(x_{1}, x_{2}, t\right)$. We apply the DRBEM to solve for $\psi$ in the square domain subject to the generated boundary data. The boundary of the square domain is divided into $N$ elements of equal length and the interior collocation points are chosen as $(m /(M+1), n /(M+1))$ for $m=1,2, \ldots, M$ and $n=1,2, \ldots, M$. As in the first problem above, we take the parameter $r$ in (14) to be $1 / 4$.

Two sets of numerical values of $T$ at the point $(0.50,0.50)$ at selected time instants are compared with the exact solution in Table II. Set A is obtained by using $(N, M)=(60,7)$ (49 interior collocation points) and $\Delta t=0.20$, whereas Set B by $(N, M)=(160,15)(225$ interior collocation points) and $\Delta t=2 / 30 \simeq 0.06667$. At a given time level, both sets of numerical values

TABLE II. Numerical and exact values of $T$ at $(0.50,0.50)$ at selected time instants.

\begin{tabular}{lcccc}
\hline Time $t$ & Set A & Set B & Azis and Clements & Exact \\
\hline 0.10 & 0.65327 & 0.64973 & 0.64907 & 0.64935 \\
0.30 & 0.54941 & 0.54946 & 0.54914 & 0.54945 \\
0.50 & 0.47550 & 0.47614 & 0.47587 & 0.47619 \\
0.70 & 0.41969 & 0.42010 & - & 0.42017 \\
0.90 & 0.37542 & 0.37585 & - & 0.37594 \\
\hline
\end{tabular}




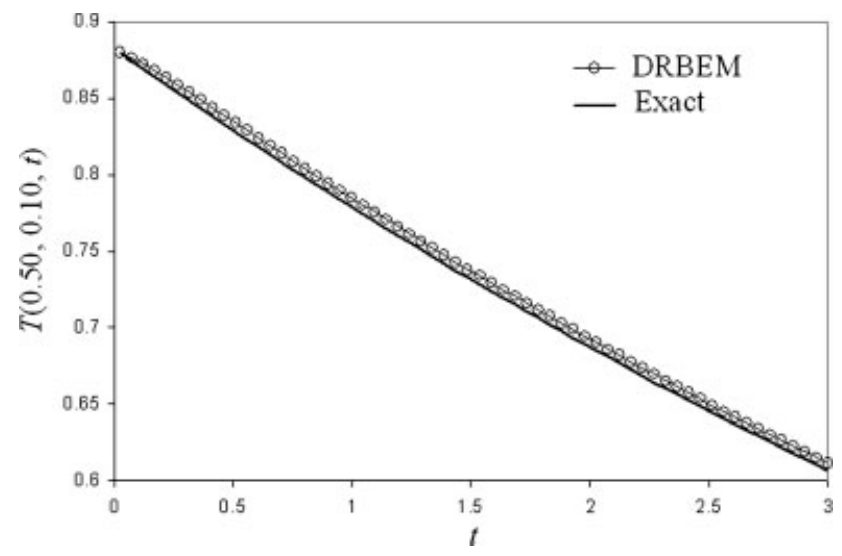

FIG. 2. A graphical comparison of the numerical and exact $T$.

are obtained after iterating 5 times to and fro the last two steps in Section IV. Set B gives more accurate numerical values of $T$ than Set $\mathrm{A}$, that is, the numerical solution shows convergence towards the exact one when the DRBEM calculation is refined. Numerical values of $T$ obtained by Azis and Clements [9] by using a very small time-step of 0.001 , discretizing the boundary into 160 boundary elements and subdividing the solution domain into 1600 cells (for treating the domain integral in their formulation) are also shown in Table II for $t=0.10,0.30$, and 0.50 . (No numerical value of $T$ is given in [9] for $t=0.70$ and $t=0.90$.) Even though a relatively large time-step is used here in Set A, the numerical values obtained appear to be quite comparable in accuracy with those of [9].

Problem 3. In the two particular problems above, the spatial function $g$ in (2) is such that the coefficient $B$ in the partial differential equation in (8) is zero. For a problem in which $B$ does not vanish in the solution domain, here we take

$$
\left[k_{i j}\right]=T \exp \left(x_{1}\right)\left[\begin{array}{ll}
1 & 1 \\
1 & 3
\end{array}\right] \text { and } \rho c=T \exp \left(x_{1}\right)
$$

so that $\lambda_{11}=1, \lambda_{12}=\lambda_{21}=1, \lambda_{22}=3, g\left(x_{1}, x_{2}\right)=\exp \left(x_{1}\right)$, and $h(T)=T$.

The solution domain is as in Problem 1 (Fig. 1), that is, $0<x_{1}<1,0<x_{2}<1 / 5$. The initial-boundary conditions are taken to be

$$
\begin{aligned}
T\left(x_{1}, x_{2}, 0\right) & =\exp \left(-\frac{1}{4} x_{1}\right) \text { for }\left(x_{1}, x_{2}\right) \in R, \\
T\left(0, x_{2}, t\right) & =\exp \left(-\frac{1}{8} t\right) \text { for } 0<x_{2}<\frac{1}{5} \text { and } t>0, \\
T\left(1, x_{2}, t\right) & =\exp \left(-\frac{1}{8} t-\frac{1}{4}\right) \text { for } 0<x_{2}<\frac{1}{5} \text { and } t>0, \\
\left.k_{i j} n_{i} \frac{\partial T}{\partial x_{j}}\right|_{x_{2}=0} & =0 \text { for } 0<x_{1}<1 \text { and } t>0, \\
\left.k_{i j} n_{i} \frac{\partial T}{\partial x_{j}}\right|_{x_{2}=1 / 5} & =0 \text { for } 0<x_{1}<1 \text { and } t>0 .
\end{aligned}
$$


To apply the DRBEM to solve for $\psi=\frac{1}{2} \exp \left(\frac{1}{2} x_{1}\right) T^{2}$, we discretize the sides of the rectangular region into 180 equal length boundary elements, employ 105 evenly spaced out collocation points in the interior of the solution domain and choose $r$ in (14) and $\Delta t$ to be $1 / 4$ and $2 / 41$, respectively.

In Fig. 2, over the time interval $0 \leq t \leq 3$, we make a graphical comparison of the numerical values of $T(0.50,0.10, t)$ with the exact solution given by $T=\exp \left(-\frac{1}{8} t-\frac{1}{4} x_{1}\right)$. The two graphs are in reasonably good agreement with each other. The percentage errors for all the numerical values of $T(0.50,0.10, t)$ over $0 \leq t \leq 3$ are less than $0.8 \%$.

\section{CONCLUSION}

The task of solving a class of two-dimensional initial-boundary value problems governed by a generalized nonlinear heat equation for nonhomogeneous anisotropic media is considered. With the aid of the Kirchhoff's transformation and an appropriate substitution of variables, the partial differential equation is recast in a form that allows the initial-boundary value problem to be formulated in terms of an integro-differential equation suitable for the development of a dualreciprocity boundary element method. A time-stepping dual-reciprocity boundary element method is presented for the numerical solution of the initial-boundary value problem. To assess the validity and accuracy of the dual-reciprocity boundary element method, it is applied to solve a few specific problems with known solutions. The numerical results obtained agree favorably with the known solutions indicating that the method can be used to provide reliable and accurate numerical solutions for the nonlinear heat equation.

\section{References}

1. J. T. Katsikadelis and M. S. Nerantzaki, The boundary element method for nonlinear problems, Eng Anal Boundary Elements 23 (1999), 365-373.

2. W. T. Ang, The two-dimensional reaction-diffusion Brusselator system: a dual-reciprocity boundary element solution, Eng Anal Boundary Elements 27 (2003), 897-903.

3. A. P. S. Selvadurai, Nonlinear mechanics of cracks subjected to indentation, Canadian J Civil Eng 33 (2006), 766-775.

4. J. T. Chen, C. C. Hsiao, Y. P. Chiu, and Y. T. Lee, Study of free surface seepage problems using hypersingular equation, Commun Numer Methods Eng 23 (2007), 755-769.

5. M. Dehghan and D. Mirzaei, The dual reciprocity boundary element method (DRBEM) for twodimensional sine-Gordon equation, Computer Methods Appl Mechanics Eng 197 (2008), 476-486.

6. M. Kikuta, H. Togoh, and M. Tanaka, Boundary element analysis of nonlinear transient heat conduction problems, Computer Methods Appl Mechanics Eng 62 (1987), 321-329.

7. T. Goto and M. Suzuki, A boundary integral equation method for nonlinear heat conduction problems with temperature-dependent material properties, Int J Heat Mass Transfer 39 (1996), 823-830.

8. D. L. Clements and W. S. Budhi, A boundary element method for the solution of a class of steady-state problems for anisotropic media, ASME J Heat Transfer 121 (1999), 462-465.

9. M. I. Azis and D. L. Clements, Nonlinear transient heat conduction problems for a class of inhomogeneous anisotropic materials by BEM, Eng Anal Boundary Elements 32 (2008), 1054-1060.

10. W. T. Ang, D. L. Clements, and N. Vahdati, A dual-reciprocity boundary element method for a class of elliptic boundary value problems for nonhomogeneous anisotropic media, Eng Anal Boundary Elements 27 (2003), 49-55. 
11. W. T. Ang, A time-stepping dual-reciprocity boundary element method for anisotropic heat diffusion subject to specification of energy, Appl Math Computation 162 (2005), 661-678.

12. W. T. Ang and K. C. Ang, A dual-reciprocity boundary element solution of a generalized non-linear Schrodinger equation, Numer Methods Partial Diff Eq 20 (2004), 843-854.

13. F. París and J. Cañas, Boundary element method: Fundamentals and applications, Oxford University Press, Oxford, 1997.

14. Y. Zhang and S. Zhu, On the choice of interpolation functions used in the dual-reciprocity boundaryelement method, Eng Anal Boundary Elements 13 (1994), 387-396. 